\title{
Design for Debate in Robotics: Questioning Paradigms in a Techno-social Discourse
}

\author{
Wouter Reeskamp and Katrien Ploegmakers \\ Department of Industrial Design, University of Technology Eindhoven, \\ P.O. Box 513, 5600 MB Eindhoven, The Netherlands \\ wouter@studiosophisti.nl, katrien@kaasdesign.nl
}

\begin{abstract}
Design for Debate' is an emerging approach in the domain of design. This paper proposes a more pragmatic application. A workshop was developed and conducted with experts in the field of social robotics. Concepts for affective robots were visualized to provoke out-of-the-box thinking. During eight workshops, robotic professionals questioned their paradigms.
\end{abstract}

Keywords: Design for Debate, Critical Design, Social Robotics, Paradigms, Design Parameters, Workshop.

\section{Introduction}

'Design for Debate' has been introduced in academic circles by Tony Dunne and Bill Gaver [1] by describing their vision of an 'artist-designer' as a creator of what they call 'value fiction'. They advocate that a critical professional can create design proposals to explore and question a two-sided story that comes along with development of a future techno-society. What they propose is design-centred fiction meant to challenge the meaning of these paradigms, and think beyond in terms of social values that are interesting to address.

'Design for Debate' hasn't been explicitly defined, but based on interviews [2] and work of Dunne [3] we derive the following aspects.

Design for Debate is a way to start discussion in society at large

Design for Debate is focussed on the meaning of future scenarios, mostly in relation with emerging technologies

Design for Debate makes use of designers' visualisation skills to make abstract concepts tangible and discussable

Design for Debate is value-centred instead of fact-centred

Discussion within Design for Debate is meant to question values of the future, but doesn't necessarily seek answers

\subsection{Applying 'Design for Debate'}

The study set-up was different compared to 'Design for Debate' in that respect it did not aim at society at large, but at a techno-social professional discourse. Fundamentally the people who are giving shape to the future techno society. Another difference was that 
we intended not only to raise questions, but also to capture given reactions. Our method therefore aims at challenging other designers to rethink the values and meanings of their visions on future society. You could say that it's less of a research tool and more a method of out-of-the-box thinking. We chose the domain of 'Affective Robotics' (also known as Personal or Social Robotics) since this field is, in terms of design and social conventions still, very much under development.

\section{Study Set-Up}

\subsection{Mapping Design Parameters}

When discussing a topic as broad as robotics in a cross-cultural setting, discussions can become too comprehensive and therefore superficial. To prevent this, we created a framework from parameters of affective robot design and herewith outlined the topics of our interest. We focused on two questions: 'What 'appearance' parameters are useful stimuli to create the notion of an affectionate experience with an object?' and 'What 'behavior' parameters are necessary to create and maintain an affectionate experience with an object?' This resulted in twelve topics (Table 1). It is not an all-embracing framework; it just contains a dozen topics we think are of importance, when developing a social robot.

We created two parameter-models (Fig. 2), one on appearance and one on behavior. Each parameter-model addresses six topics. To stage a discussion about the twelve topics we defined scales ranging from one extreme to another.

Table 1. The twelve design parameters

\begin{tabular}{l|l}
\hline Appearance & Behavior \\
\hline Shape & Obedience \\
Tactility & Functionality \\
Face & Learning capabilities \\
Body language & Specialization \\
Sound & Intelligence \\
Temperature & Stickiness \\
\hline
\end{tabular}

\subsection{Developing Provoking Concepts}

To have the right type of discussion with professionals, it is crucial to let them think beyond paradigms familiar to them in their everyday practice. The approach we propose with 'Design for Debate' is to challenge the participants with extreme, thought-provoking concepts to bring visions and relations out into the open. In our case we proposed six affective robots in scenarios, all technical and social more or less feasible, but still ambivalent in their characteristics.

An important premise is the mixture of extremes to make sure that discussion is not dogmatically heading in one direction. We used two (physical) parameter-models systematically to generate the design proposals. This interlinked the parameters with the concepts used to provoke the discussion. (Fig.1). 

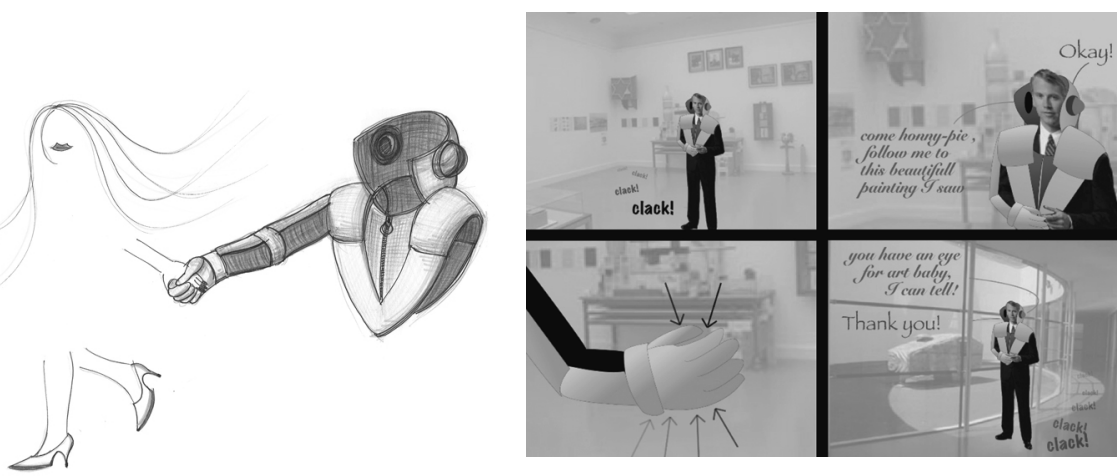

Fig. 1. Babe-bot concept: When wearing the special suit, a beautiful lady/man, whom you cannot see but can clearly hear and feel, will guide you through the museum

\subsection{Workshop Participants}

As input for our design-research we staged a debate with Dutch and Japanese parties involved in research, development and design of robots for affection. Eight workshops were done to make this (virtual) debate possible. The participants were industrial companies including Sony, Philips Research and R2R, academic institutes like the Robotics Department of Osaka University, Nirvana Technologies, ATR, AIST and the Designed Intelligence group of the University of Technology Eindhoven.

\subsection{Chair Discussions}

From each concept product-drawings and a user-scenario were shown and the parameter-models were set according to the design proposal. The models were used after each concept presentation to provoke a discussion with the participants (Fig.2), by asking questions such as: 'What parameter do you think makes this a strong concept?' and 'which parameter would you suggest to change in order to create a robot that is more affective?' The aim was not to assess the designs as such, yet to trigger the developers to think and react on possibilities in the field of robotics beyond those they are familiar with.



Fig. 2. Workshop participants (Japanese and Dutch) adjust the parameter-models 


\section{Conclusion}

Since we had eight sessions to compare our study in 'Design for Debate', a solid idea of its value has arisen. Our approach in 'Design for Debate' proved to be of great value in pinpointing the main assumptions, visions and paradigms in a discourse of 'Affective Robotics'. E.g. a senior researcher said in discussion on the 'Mystic-bot' "A ball as a robot? Before this workshop I didn't think a ball could be a robot". Or when a participant claimed regarding the 'Babe-bot': "An affective robot should be physical", but during the discussion this was questioned. "You can have affection for a person you met in the virtual world of internet, so why not for a virtual robot?" (Fig.1) As a research tool it proved to be more difficult, since opinions tend to be too varied to draw conclusions. A better opportunity is that 'Design for Debate' offers a starting point for a creative process. It can serve as a 'vehicle of communication'; it sets certain definitions and it sparks imagination. And since it makes use of knowledge and wisdom of people it is less time-consuming then literature or scientific research, without leading to superficial results. But due to the subjective nature it is simply not appropriate for reproducible study. 'Design for Debate' cán be of value to set an agenda for these research topics.

\section{Discussion}

Currently discussion on 'Design for Debate' is mainly arising in the discourse of design research, though we foresee its value in commercial context as well. Due to its practical nature, orientating research can be done in a relative short time-span. Moreover the results are often inspiring and can be a catalyst in following creative processes. Due to its open yet focused principle we think it is especially relevant to address complex problems where social, technological, psychological and economic factors are intertwined.

\section{References}

1. Dunne, A., Gaver, W.: The Pillow: Artist-Designers in the Digital Age. In: CHI97 (1997)

2. Debatty, R.: Interview with Anthony Dunne (2007), http://www.we-make-money-not-art.com

3. Dunne, A., Raby, F.: Design Noir: The secret life of electronic objects, August BirkHauser (2001) 